DIVISION OF THE HUMANITIES AND SOCIAL SCIENCES

CALIFORNIA INSTITUTE OF TECHNOLOGY

PASADENA, CALIFORNIA 91125

A SUBJECTIVE SPIN ON ROULETTE WHEELS

Paolo Ghirardato

California Institute of Technology and ICER (Torino)

Fabio Maccheroni

Università Bocconi and ICER (Torino)

Massimo Marinacci

Università di Torino and ICER (Torino)

Marciano Siniscalchi

Princeton University

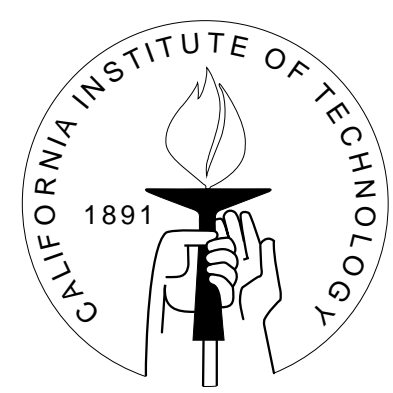

SOCIAL SCIENCE WORKING PAPER 1127 


\title{
A Subjective Spin on Roulette Wheels
}

\author{
Paolo Ghirardato $\quad$ Fabio Maccheroni Massimo Marinacci \\ Marciano Siniscalchi
}

\begin{abstract}
We provide a behavioral foundation to the notion of 'mixture' of acts, which is used to great advantage in the decision setting introduced by Anscombe and Aumann [1].

Our construction allows one to formulate mixture-space axioms even in a fully subjective setting, without assuming the existence of randomizing devices. This simplifies the task of developing axiomatic models which only use behavioral data. Moreover, it is immune from the difficulty that agents may 'distort' the probabilities associated with randomizing devices.

For illustration, we present simple subjective axiomatizations of some models of choice under uncertainty, including the maxmin expected utility model of Gilboa and Schmeidler [11], and Bewley's model of choice with incomplete preferences [2].

JEL classification numbers: D81
\end{abstract}

Key words: Roulette Wheels, Subjective Mixtures, Mixture Space, Maxmin Expected Utility, Incomplete Preferences 


\title{
A Subjective Spin on Roulette Wheels*
}

\author{
Paolo Ghirardato $\quad$ Fabio Maccheroni Massimo Marinacci \\ Marciano Siniscalchi
}

\section{Introduction}

The axiomatizations of subjective expected utility (SEU) of Savage [18] and Anscombe and Aumann [1, AA for short] are often contrasted in terms of their analytical complexity and behavioral content.

In particular, Savage explicitly aimed at constructing a theory that relies solely upon purely behavioral data - preferences among acts, i.e. maps assigning consequences to states. In contrast, the AA approach requires assuming the existence of pre-assigned, 'objective' probabilities. However, the latter is much more amenable to mathematical treatment than Savage's approach, as it allows for a direct application of familiar vector space techniques.

The main contribution of this paper is to show that it is possible to reconstruct the AA setting in a fully behavioral fashion; this places models that follow the AA approach on comparable epistemological footing with those that follow Savage's, at virtually no cost in terms of analytical tractability.

As noted above, Savage's purpose was to show that subjective probability and utility can be derived without assuming the existence of extraneous devices characterized by 'objective' probabilities, or 'ethically neutral' events. This independence from nonbehavioral data makes it relatively easy to test the assumptions and implications of the theory; arguably, such features have been instrumental in making SEU maximization the reference model in economic theory.

*An earlier version of this paper was circulated with the title "Subjective Foundations for Objective Randomization: A New Spin on Roulette Wheels". We thank Mark Machina and the audiences at the V SAET Conference (Ischia, July 2001) and the RUD 2001 Conference (Venezia, July 2001) for helpful comments. Maccheroni thanks the Department of Economics at Boston University and the Division of HSS at Caltech, while Siniscalchi thanks the MEDS Department at the Kellogg Graduate School of Management (Northwestern University), for their hospitality while this paper was being written. The authors' e-mail addresses are respectively: paolo@hss.caltech.edu; fabio.maccheroni@uni-bocconi.it; massimo@econ.unito.it; marciano@princeton.edu. 
Anscombe and Aumann suggested abandoning Savage's general setting and allowing payoffs to be lotteries contingent on the behavior of a randomizing device, which they called a 'roulette wheel'. They observed that the roulette wheel enables one to define 'objective mixtures' of acts, and hence to extend the axiomatics of von Neumann and Morgenstern [21] to the case in which the probabilities of the (non-roulette) events are not objective.

This considerably simplifies the axiomatic derivation of the SEU model. The presence of mixtures makes the set of acts a convex subset of a vector space: If the decision maker's preferences conform to expected utility over the 'roulette wheel acts', ${ }^{1}$ the utility profile corresponding to the 'objective mixture' of two acts is the convex combination of the utility profiles of the latter. Such algebraic structure may be exploited in the formulation of the axioms, as well as in the construction of the mathematical representation.

Appropriate extensions of the AA setting also simplify the development of models that address well-known descriptive limitations of the SEU representation (see, e.g, Luce [13, Chap. 3]). Many of the most successful extensions of SEU were first carried out in an AA-type setting: among others, we mention Schmeidler's 'Choquet expected utility' (CEU) model [19], Gilboa and Schmeidler's 'maxmin expected utility' (MEU) model [11], and Bewley's model [2] of choice with incomplete preferences. ${ }^{2}$

However, by employing a roulette wheel, the AA approach reintroduces a non-behavioral element in the decision model, thus restricting its conceptual appeal and its scope (see our discussion of 'distorted probabilities' below).

In this paper, we show that it is possible to construct a 'subjective AA setting' in a fully behavioral setting like Savage's, thus overcoming this difficulty. Our construction holds for preferences that satisfy some mild conditions, and it requires that the set of possible outcomes be sufficiently rich (e.g., an interval in the real line). If these conditions are met, 'subjective mixtures' of acts can be constructed using only behavioral data. More details on this construction are given below.

As an illustration of our technique, we employ subjective mixtures to offer simple axiomatizations of the CEU and MEU models (of which SEU is a special case), as well as of Bewley's model. Essentially, our main result allows us to state and characterize axioms as if the analysis was carried out in the AA setting. However, we do not need roulette wheels. Clearly, other fruitful applications of our subjective AA setting are possible.

Identifying a subjective vector structure in Savage's setting is not merely of 'technical' interest. In fact, the subjective construction has at least two significant conceptual advantages. First, it enables us to avoid the philosophically difficult concept of 'objective'

\footnotetext{
${ }^{1}$ The acts which only depend on the behavior of the roulette wheel.

${ }^{2}$ Some of these models have later been reformulated in Savage-type settings; however, this line of research has clearly shown that the 'purity' of Savage's approach imposes substantial costs in terms of analytical complexity.
} 
probability. As de Finetti and Savage argued extensively, the existence of objective probabilities is debatable.

Second, it sidesteps a descriptive limitation of the AA framework. It is well known that many experimental subjects do not act consistently with the expected utility model even when choosing among roulette wheel acts: they behave as if they 'distorted' the probabilities of the roulette events. Consequently, these subjects do not treat the set of acts with objective mixtures as a convex subset of a vector space, and the axioms in the style of von Neumann and Morgenstern become meaningless for them. Thus, the advantage of the AA setting effectively disappears.

This is not the case with subjective mixtures, as the notion of subjective mixture is unrelated to external objects, which could be interpreted differently by the decision maker and the modeller. For any preference relation that satisfies our minimal conditions, the subjective vector structure of the set of acts can be observed by the modeller.

\section{Preference Averages and Subjective Mixtures of Acts}

The key step in our construction of subjective mixtures of acts is the notion of a "preference average' of two outcomes. In our interpretation, a preference average of two outcomes $x$ and $y$ is an outcome $z$ with the following property: if $x$ and $y$ are possible results which play a symmetric role in the decision maker's evaluation of a bet, then replacing $x$ and $y$ with $z$ leaves the decision maker indifferent. This appears to us to constitute a sensible behavioral definition of the midpoint of the preference interval between two outcomes.

To see how a preference average is practically observed, fix an event $E$, and consider the decision maker's preferences over the bets on $E$, i.e., uncertain prospects of the form

$x E y=$ 'receive $x$ if $E$ obtains, and $y$ otherwise', where outcome $x$ is (weakly) preferred to outcome $y$. Take $E$ to be 'non-trivial'; that is, for some pair $x$ and $y$ such that $x$ is strictly preferred to $y$ the bet $x E y$ is strictly between $x$ and $y$. Also, assume that every $x E y$ has a certainty equivalent $c_{x E y}$.

Given two such outcomes $x$ and $y$, suppose that $z$ is a third outcome that satisfies the following condition:

$$
x E y \text { is indifferent to } c_{x E z} E c_{z E y} \text {. }
$$

Under mild assumptions on the decision maker's preference, such a $z$ exists for every $x$ and $y$. We now argue that the same assumptions allow us to interpret $z$ as a 'preference average' of $x$ and $y$. 
Since $x E y$ is trivially indifferent to $c_{x E x} E c_{y E y}$, we have that

$$
c_{x E x} E c_{y E y} \text { is indifferent to } c_{x E z} E c_{z E y} \text {. }
$$

Substituting $z$ for the inner $x$ and $y$ in the 'compound' act on the left-hand side leaves the decision maker indifferent; thus, $z$ is a 'local' certainty equivalent for $x$ and $y .^{3}$

Moreover, our preference axioms imply that the inner outcomes in the 'compound' bets in Eq. (2) have the same impact on the decision maker's evaluation. Formally, if $z^{\prime}$ and $z^{\prime \prime}$ are both between $x$ and $y$ in preference, then

$$
c_{x E z^{\prime}} E c_{z^{\prime \prime} E y} \text { is indifferent to } c_{x E z^{\prime \prime}} E c_{z^{\prime} E y} \text {. }
$$

In words, permuting $z^{\prime}$ and $z^{\prime \prime}$ does not affect the decision maker's preferences: the two inner outcomes play a symmetric role in his evaluation of these bets.

We conclude that $z$ is a local certainty equivalent for $x$ and $y$ in a situation in which $x$ and $y$ have the same impact on the decision maker's preferences over bets on the same event $E$. Therefore, $z$ fits our intuitive description of a preference average of $x$ and $y$.

To see this from a different perspective, we remark that the same mild assumptions on preferences imply that there exists a cardinal utility $u$ on the set of outcomes and a number $\rho(E) \in(0,1)$ such that $x E y$ is preferred to $x^{\prime} E y^{\prime}$ if and only if ${ }^{4}$

$$
u(x) \rho(E)+u(y)(1-\rho(E)) \geq u\left(x^{\prime}\right) \rho(E)+u\left(y^{\prime}\right)(1-\rho(E)) .
$$

Using this representation, it is simple to show that $z$ satisfies Eq. (1) if and only if

$$
u(z)=\frac{1}{2} u(x)+\frac{1}{2} u(y) .
$$

The key step in the proof is the quantitative counterpart to the intuition provided above: The inner outcomes $x$ and $y$ in the act $c_{x E x} E c_{y E y}$ and the $z$ 's in the act $c_{x E z} E c_{z E y}$ all receive the same 'weight' according the evaluation functional described by Eq. (3). (Notice that Eq. (4) also implies that the notion of preference average is independent of the 'non-trivial' event $E$ that we use to construct it.)

The preference average described above corresponds to the $\frac{1}{2}: \frac{1}{2}$ mixture of two outcomes. It is clear that by iterating our definition, arbitrary dyadic mixtures may be defined. Finally, we can use these dyadic mixtures of outcomes to define subjective mixtures of acts state by state, as in the AA framework.

\footnotetext{
${ }^{3}$ This terminology is justified by noting that $v$ is a (global) certainty equivalent of $w E w^{\prime}$ if $v E v$ is indifferent to $w E w^{\prime}$.

${ }^{4}$ We emphasize that the following representation only holds for bets on $E$ (acts of the form $x E y$ with $x$ (weakly) preferred to $y$ ). As the results in Sections 3 and 4 indicate, our axioms allow considerable flexibility in the evaluation of all other acts.
} 


\section{Related Literature and Organization of the Paper}

This paper is not first in remarking the advantages of identifying an algebraic structure in the choice set. A few other papers have attempted to build a vector structure in Savage's setting. Most notable is the work of Gul [12] (later extended by Chew and Karni [5] and Casadesus-Masanell, Klibanoff and Ozdenoren [4]). His main objective is to provide an axiomatization of SEU which, differently from Savage's, does not require the state space to be infinite. As we do, he requires that the set of outcomes be infinite and that every bet have a certainty equivalent. He then assumes the existence of an 'ethically neutral' event $A$ such that the preference over bets on $A$ have an SEU representation assigning probability $1 / 2$ to it. Then, it is natural to call a certainty equivalent of the bet $x A y$ the 'eventwise' preference average of $x$ and $y$.

The major difference with Gul's paper lies in the use of the notion of preference average. Gul employs it as a technical device to derive a cardinal utility function over outcomes, which is then used to explicitly construct probabilities and show that preferences admit a SEU representation. In contrast, we show that it can be used to endow the set of acts with an AA-style algebraic structure. As a result, the usual axiomatics and functional-analytic machinery deliver the characterization results with minimal effort (cf. Prop. 9).

There is also a significant difference between the two notions of preference average. Suppose that we need to observe the preference average of two outcomes $x$ and $y$ for two decision makers, one satisfying our model and one satisfying Gul's. For the former, we apply the condition explained above using some 'non-trivial' event $E$. For the latter, it is necessary to find an event $A$ such that the decision maker satisfies SEU over all the bets on $A$. Since the elicitation of such event $A$ involves the observation of infinitely many comparisons, our construction of preference averages is clearly more direct than that of eventwise preference averages, therefore much easier to interpret and implement.

As to the extensions of Gul's work, Chew and Karni [5] show that assuming that the bets on $A$ satisfy SEU and that $A$ is 'ethically neutral' is not necessary to obtain a SEU (and CEU) representation. Such generalization prevents the construction of a vector space structure over the set of acts, ${ }^{5}$ which is anyway not an objective of their work.

In an axiomatization of the MEU model that generalizes Gul's, Casadesus-Masanell et al. [4] assume that the bets on $A$ satisfy SEU (but not that $A$ is ethically neutral). They do not suggest iterating eventwise preference averages to induce an algebraic structure over acts. On the other hand, in the companion paper [3] Casadesus-Masanell et al. do explicitly attempt to build a vector structure in Savage's setting, in order to retain the spirit of the axiomatization of MEU in the AA setting. Their notion of subjective mixtures is based on standard sequences, and is therefore less immediate than the one we present. The same is said of the relative interpretations of the axioms.

\footnotetext{
${ }^{5}$ If $f$ and $g$ do not dominate each other statewise, there are $s$ and $s^{\prime}$ such that $f(s) A g(s)$ and $f\left(s^{\prime}\right) A g\left(s^{\prime}\right)$ are bets on different events. Intuitively: we are mixing with different weights in different states.
} 
Machina [14] moves along a different direction in showing how to construct 'almostobjective' events in a Savage-style setting. He assumes that the state space has a Euclidean structure, and that preferences satisfy an 'event smoothness' condition; he then constructs sequences of events that, in the limit, are treated by the decision-maker 'as if' they had an 'objective' (agreed upon) probability. He investigates the properties of almost-objective events, and also suggests constructing almost-objective mixtures of acts. ${ }^{6}$ The latter differ from our subjective mixtures, in that they do not yield a preference average of the outcomes in every state, and do not induce an algebraic structure over the set of acts.

A secondary contribution of this paper is the introduction of simple axiomatic characterizations of the CEU, MEU, and Bewley's models in Savage's setting. To the best of our knowledge, there are no axiomatizations of the latter in Savage's setting. There exist several axiomatizations of the CEU model in Savage's setting (e.g., Gilboa [10], Nakamura [16] and Chew and Karni [5]), while Casadesus-Masanell et al. [3, 4] contain the only axiomatizations of MEU. The main advantage of our characterizations lies in their simplicity.

Finally, this paper improves over Ghirardato and Marinacci [9] by showing how to refine their 'biseparable preferences' model in order to guarantee the uniqueness of what they call 'the canonical functional' (see also Ghirardato, Maccheroni and Marinacci [7]).

The paper proceeds as follows. Section 1 introduces the required terminology and notation. Section 2 is the core of the paper. It introduces the notion of subjective mixture formally, as well as the preference conditions that are needed to make it consistent. Section 3 presents the axiomatizations of the CEU, SEU and MEU models, while Section 4 looks at Bewley's model. The appendices contain the proofs of the results in the main text, as well as some more technical material.

\section{Preliminaries}

Consider a set $S$ of states of the world, an algebra $\Sigma$ of subsets of $S$ called events, and a set $X$ of consequences. We denote by $\mathcal{F}$ the set of all the simple acts: finite-valued functions $f: S \rightarrow X$ which are measurable with respect to $\Sigma$. For $x \in X$ we define $x \in \mathcal{F}$ to be the constant act such that $x(s)=x$ for all $s \in S$. So, with the usual slight abuse of notation, we identify $X$ with the subset of the constant acts in $\mathcal{F}$. Also, given $x, y \in X$ and $A \in \Sigma$, we use $x A y$ to denote the binary act which yields $x$ if $s \in A$ and $y$ otherwise.

We model the DM's preferences on $\mathcal{F}$ by a binary relation $\succcurlyeq$. A functional $V: \mathcal{F} \rightarrow \mathbb{R}$ represents $\succcurlyeq$ if $V(f) \geq V(g)$ if and only if $f \succcurlyeq g$. Clearly, a necessary condition for $\succcurlyeq$ to have a representation is that it be a weak order - a complete and transitive relation

\footnotetext{
${ }^{6}$ For instance, an almost-objective mixture of two constant acts $x, y$ is defined as a bet $x E y$ on the appropriate almost-objective event $E$.
} 
- so that, as customary, we can denote by $\sim$ and $\succ$ its symmetric and asymmetric components, respectively. $V$ is monotonic if $V(f) \geq V(g)$ whenever $f(s) \succcurlyeq g(s)$ for all $s \in S$.

We let $B(\Sigma)$ denote the set of all real-valued $\Sigma$-measurable simple functions. Given a functional $I: B(\Sigma) \rightarrow \mathbb{R}$, we say that $I$ is: monotonic if $\varphi \geq \psi$ implies $I(\varphi) \geq I(\psi)$ for all $\varphi, \psi \in B(\Sigma)$; c-linear if $I(a \varphi+b)=a I(\varphi)+b$ for all $\varphi \in B(\Sigma)$, all $a \in \mathbb{R}$ such that $a \geq 0$, and all $b \in \mathbb{R}$.

Next, we need to define a special class of functions on $\Sigma$. A set-function $\rho$ on $(S, \Sigma)$ is called a capacity if it is monotone and normalized; that is, $\rho(A) \leq \rho(B)$ if $A \subseteq B$, and $\rho(\emptyset)=0$ and $\rho(S)=1$. A capacity is called a probability if it is (finitely) additive: $\rho(A \cup B)=\rho(A)+\rho(B)$ if $A \cap B=\emptyset$.

Finally, we have an assumption on the set of consequences that holds except where otherwise noted.

Structural Assumption The set $X$ is a connected and separable topological space with topology $\tau$.

\section{Preferences with Subjective Mixtures}

In this section we provide a basic characterization result for a general class of preferences that includes most of the decision models originally formulated in an AA framework. The axioms guarantee that the preferences have enough structure to define a 'subjective mixture' operation that is the subjective equivalent of the 'objective mixture' in the AA framework.

\subsection{Basic Axioms}

The first two axioms in the characterization are standard. First of all, we require that the relation be a weak order.

Axiom A 1 (Weak Order) (a) For all $f, g \in \mathcal{F}, f \succcurlyeq g$ or $g \succcurlyeq f$. (b) For all $f, g, h \in$ $\mathcal{F}$, if $f \succcurlyeq g$ and $g \succcurlyeq h$, then $f \succcurlyeq h$.

Next, we require that the preference satisfy the behavioral equivalent of monotonicity.

Axiom A 2 (Dominance) For every $f, g \in \mathcal{F}$, if $f(s) \succcurlyeq g(s)$ for every $s \in S$ then $f \succcurlyeq g$. 
The next axiom requires a definition. We say that an event $A \in \Sigma$ is essential if $x \succ x A y \succ y$ for some consequences $x$ and $y$ such that $x \succ y$.

Axiom A 3 (Essentiality) There exists an essential event $E \in \Sigma$.

This axiom implies that $\succcurlyeq$ is nontrivial, as no event is essential if $\succcurlyeq$ is trivial. Given $E$, we denote by $\Sigma_{E}$ the algebra generated by $E$ and by $\mathcal{F}_{E}$ the set of the $\Sigma_{E}$-measurable acts.

We now have three axioms that impose with one exception restrictions on the DM's preferences over the acts in $\mathcal{F}_{E}$. The first (a weak version of Savage's P3 [18] axiom) is a converse to axiom A2. We use the following terminology: An event $A \in \Sigma$ is null (resp. universal) if $y \sim x A y$ (resp. $x \sim x A y$ ) for every $x \succ y$. It follows from axiom A1 that an event can be only one of null, essential, or universal.

Axiom A 4 (E-Monotonicity) For every non-null $A \in \Sigma_{E}$ and every $x, y \succcurlyeq z \in X$,

$$
x \succ y \Longrightarrow x A z \succ y A z .
$$

For every non-universal $A \in \Sigma_{E}$ and every $x, y \preccurlyeq z \in X$,

$$
x \succ y \Longrightarrow z A x \succ z A y
$$

The next axiom is a weak continuity property. To state it, we first observe that the topology $\tau$ on $X$ induces the product topology on the set $X^{S}$ of all functions from $S$ into $X$. In this topology, a net $\left\{f_{\alpha}\right\}_{\alpha \in D} \subseteq X^{S}$ converges to $f \in X^{S}$ if and only if $f_{\alpha}(s) \stackrel{\tau}{\longrightarrow} f(s)$ for all $s \in S$ (remember that $S$ is arbitrary). For this reason it is also called the topology of pointwise convergence.

Axiom A 5 (E-Continuity) Let $\left\{g_{\alpha}\right\}_{\alpha \in D} \subseteq \mathcal{F}_{E}$ be a net that pointwise converges to g. For every $f \in \mathcal{F}$, if $g_{\alpha} \succcurlyeq f$ (resp. $f \succcurlyeq g_{\alpha}$ ) for all $\alpha \in D$, then $g \succcurlyeq f$ (resp. $f \succcurlyeq g$ ).

This axiom is the announced exception, as the act $f$ can be any element of $\mathcal{F}$. It is straightforward to show (see Lemma 12 in Appendix B) that any binary relation satisfying axioms A1-A3 and A5 (on a connected $X$ ) has certainty equivalents. That is, for every $f \in \mathcal{F}$, there exists a $x \in X$ such that $x \sim f$. Granted this, we henceforth denote by $c_{f}$ an arbitrarily chosen certainty equivalent of $f \in \mathcal{F}$.

The next axiom imposes a behavioral restriction. We write $x \succcurlyeq\left\{z^{\prime}, z^{\prime \prime}\right\}$ (resp. $\left.\left\{z^{\prime}, z^{\prime \prime}\right\} \succcurlyeq y\right)$ if $x \succcurlyeq z^{\prime}$ and $x \succcurlyeq z^{\prime \prime}\left(\operatorname{resp} . z^{\prime} \succcurlyeq y\right.$ and $\left.z^{\prime \prime} \succcurlyeq y\right)$

Axiom A 6 (E-Substitution) For all $x, y, z^{\prime}, z^{\prime \prime} \in X$ and $A, B \in \Sigma_{E}$. Suppose that $x \succcurlyeq\left\{z^{\prime}, z^{\prime \prime}\right\} \succcurlyeq y$. Then

$$
c_{x A z^{\prime}} B c_{z^{\prime \prime} A y} \sim c_{x B z^{\prime \prime}} A c_{z^{\prime} B y} .
$$




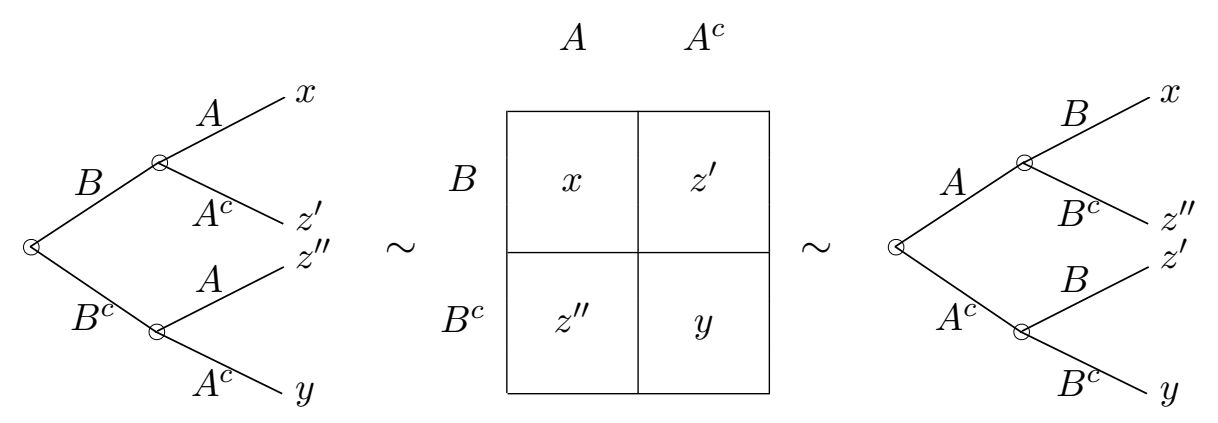

Figure 1: The acts $c_{x} A z^{\prime} B c_{z^{\prime \prime} A y}$ and $c_{x B z^{\prime \prime}} A c_{z^{\prime} B y}$

Axioms in the spirit of our A6 are standard in the literature originating from Nakamura [16]. Note however that axiom A6 only involves preferences on the (binary) acts in $\mathcal{F}_{E} \cdot{ }^{7}$

First, Axiom A6 may be interpreted as imposing a 'substitution' requirement (see Quiggin [17] for a similar interpretation of a related axiom). Recall that, following Gul [12] and Chew and Karni [5], binary acts and their certainty equivalents may be employed to construct statewise mixtures of acts. In this sense, the act $c_{x} A z^{\prime} B c_{z^{\prime \prime}} A y$ may be regarded as the 'A-mixture' of the acts $x B z^{\prime \prime}$ and $z^{\prime} B y$. On the other hand, $c_{x B} z^{\prime \prime} A c_{z^{\prime}} B y$ is the 'A-mixture' of the certainty equivalents of the same acts. Therefore, Eq. (5) requires that substituting acts in a statewise mixture with their certainty equivalents leave the decision maker indifferent.

An alternative interpretation of the axiom involves the idea of 'reduction of compound acts' (see also Luce [13, Ch. 3]). Suppose that, in order to simplify the evaluation of 'compound' acts such as $c_{x} A z^{\prime} B c_{z^{\prime \prime}} A y$, the decision maker imagines a fictitious setting wherein two identical copies of the experiment described by $\Sigma_{E}$ are performed simultaneously. ${ }^{8}$ He then reduces these acts to fictitious 'product' acts. In particular, he reduces $c_{x A z^{\prime}} B c_{z^{\prime \prime} A y}$ (resp. $c_{x B z^{\prime \prime}} A c_{z^{\prime} B y}$ ) in the original setup to the fictitious act represented on the right (resp. on the left) of Figure 1. By the simultaneity assumption, both ficticious acts can be represented by the table in the center. Therefore, the decision maker should be indifferent between $c_{x A z^{\prime}} B c_{z^{\prime \prime} A y}$ and $c_{x B z^{\prime \prime}} A c_{z^{\prime} B y}$. Thus, axiom A6 reflects one consequence of the assumption that the decision maker adopts the evaluation procedure just described. We now show that a preference $\succcurlyeq$ that satisfies axioms A1-A6 can be given a representation $V$ which on the set $\mathcal{F}_{E}$ has a simple separable form (a weaker form of the biseparable representation introduced in [9]).

Recalling the discussion in the Introduction, Axiom A6 is responsible for the fact that the 'internal' outcomes $z^{\prime}$ and $z^{\prime \prime}$ have symmetric importance in the decision maker's evaluation of composite acts in Eq. (5).

\footnotetext{
${ }^{7}$ Chew and Karni [5] show that this axiom is equivalent to a form of comonotonic independence (cf. axiom A9 below and axiom S2 in Ghirardato and Marinacci [9]) restricted to acts in $\mathcal{F}_{E}$.

${ }^{8}$ Simultaneity reflects the idea that in the decision maker's mind the outcome of one experiment does not influence the outcome of the other in any way.
} 
The axioms stated so far imply that the DM's preferences over consequences have a cardinal representation. However, we cannot say anything (beyond monotonicity) about the structure of the functional $V$ for acts which are not $\Sigma_{E}$-measurable.

Lemma $1 \succcurlyeq$ is a binary relation satisfying axioms $A 1-A 6$ if and only if there is a $\tau$ continuous nonconstant utility index $u: X \rightarrow \mathbb{R}$ and a capacity $\rho_{E}: \Sigma_{E} \rightarrow[0,1]$, with $\rho_{E}(E) \in(0,1)$, such that the functional $V: \mathcal{F} \rightarrow \mathbb{R}$ defined by $V(f) \equiv u\left(c_{f}\right)$ for any $f \in \mathcal{F}$ represents $\succcurlyeq$, it is monotonic and it satisfies, for all $x \succcurlyeq y$ and all $A \in \Sigma_{E}$,

$$
V(x A y)=u(x) \rho_{E}(A)+u(y)\left(1-\rho_{E}(A)\right) .
$$

Moreover, such $u$ and $V$ are unique up to a positive affine transformation and $\rho_{E}$ is unique.

We stress that in general $\rho_{E}(E)+\rho_{E}\left(E^{c}\right) \neq 1$; that is, the representation of the DM's preferences over bets on events in $\Sigma_{E}$ is not necessarily expected utility with 'beliefs' $\rho_{E}$.

\subsection{Subjective Mixtures and Independence}

We now introduce the key notion of the 'subjective mixture' of two acts using the essential event $E$ of axiom A3. This is then used to state the last axiom, which guarantees that this notion of mixture is independent of the essential event $E$. We start with the definition of preference average of two consequences.

Definition 2 Given $x, y \in X$ such that $x \succcurlyeq y$ (resp. $y \succcurlyeq x)$, we denote by $(1 / 2) x \oplus$ $(1 / 2)$ y a consequence $z \in X$ such that $x \succcurlyeq z \succcurlyeq y$ (resp. $y \succcurlyeq z \succcurlyeq x$ ) and

$$
x E y \sim c_{x E z} E c_{z E y}\left(\text { resp. yE } x \sim c_{y E z} E c_{z E x}\right) .
$$

Two remarks are in order. The first is that the definition implies that $(1 / 2) x \oplus$ $(1 / 2) y \sim(1 / 2) y \oplus(1 / 2) x$. The second is that there may be many consequences that satisfy the required conditions: Lemma 3 below shows that all the consequences that satisfy the conditions form an indifference class of $\succcurlyeq$. As stated in the definition, $(1 / 2) x \oplus$ $(1 / 2) y$ denotes a representative of the indifference class. Nothing would change in our results if we required every property related to mixtures to hold for every $z$ that satisfies the conditions in Definition 2.

We explained in the Introduction why we interpret the consequence $(1 / 2) x \oplus(1 / 2) y$ as the preference average of $x$ and $y$. We now show that the consequence $z$ averages the utilities of $x$ and $y$ with the cardinal utility index $u$ provided by Lemma 1.

Lemma 3 Suppose that $\succcurlyeq$ satisfies axioms $A 1-A 6$. For any $x, y \in X$, there exists a $z=(1 / 2) x \oplus(1 / 2) y$. Moreover, if $u$ is the cardinal utility that represents $\succcurlyeq$ by Lemma 1 , $z$ is an arbitrarily chosen element of the set of the $z^{\prime}$ satisfying

$$
u\left(z^{\prime}\right)=\frac{1}{2} u(x)+\frac{1}{2} u(y)
$$


Clearly, we can consider iterated averages such as $(1 / 2) x \oplus(1 / 2)[(1 / 2) x \oplus(1 / 2) y]$, which is tantamount to a $3 / 4: 1 / 4$ mixture of $x$ and $y$. More generally, consider any dyadic rational: a number $\gamma \in(0,1)$ such that for some finite $N$,

$$
\gamma=\sum_{i=1}^{N} a_{i} / 2^{i},
$$

where $a_{i} \in\{0,1\}$ for every $i$ and $a_{N}=1$. We use $\gamma x \oplus(1-\gamma) y$ as a short-hand for the iterated preference average

$$
\frac{1}{2} z_{1} \oplus \frac{1}{2}\left(\ldots\left(\frac{1}{2} z_{N-1} \oplus \frac{1}{2}\left(\frac{1}{2} z_{N} \oplus \frac{1}{2} y\right)\right) \ldots\right),
$$

where for every $i, z_{i}=x$ if $a_{i}=1$ and $z_{i}=y$ otherwise. The mixture of acts $f$ and $g$ is now defined as the act which state by state pays the mixture of the payoffs of $f$ and $g$.

Definition 4 Given $f, g \in \mathcal{F}$ and a dyadic rational $\gamma$, we define $\gamma f \oplus(1-\gamma) g$ the act $h \in \mathcal{F}$ such that $h(s)=\gamma f(s) \oplus(1-\gamma) g(s)$ for every $s \in S$.

It follows from this definition (see Lemma 13 in Appendix B) that for every $f, g \in \mathcal{F}$ and $s \in S$,

$$
u[(\gamma f \oplus(1-\gamma) g)(s)]=\gamma u(f(s))+(1-\gamma) u(g(s)) .
$$

That is, the act $\gamma f \oplus(1-\gamma) g$ is the act whose utility profile mixes with weight $\gamma$ those of $f$ and $g$. This act has a utility profile which is identical to that of the 'objectively mixed' act $\gamma f+(1-\gamma) g$ in the AA framework. The difference is that in our framework the mixture has the correct convex combination of utilities by definition, rather than by assumption.

Our final axiom uses the notion of mixture thus derived to impose a very weak and natural property of separability of preferences.

Axiom A 7 (Weak Certainty Independence) For every $f, g \in \mathcal{F}, x \in X$ and dyadic rational $\gamma$,

$$
f \sim g \Longrightarrow \gamma f \oplus(1-\gamma) x \sim \gamma g \oplus(1-\gamma) x
$$

This axiom is a (weaker) 'subjective' version of the identically named axiom of Gilboa and Schmeidler [11]. The interpretation is analogous: If the DM prefers $f$ to $g$, he should also prefer the mixture of $f$ with the constant $x$ to the mixture of $g$ with the $x$. In fact, a mixture with a constant delivers an act whose utility profile is a positive affine transformation of the original one. The novelty in our axiom is that the mixtures used in (8) are derived in a purely subjective fashion, so that as explained above they certainly induce the appropriate profile of utilities.

The following result characterizes the preferences that satisfy the given axioms: 
Theorem 5 Let $\succcurlyeq$ be a binary relation on $\mathcal{F}$. Then $\succcurlyeq$ satisfies axioms A1-A7 if and only if there exist a $\tau$-continuous nonconstant function $u: X \rightarrow \mathbb{R}$ and a monotonic $c$-linear functional $I: B(\Sigma) \rightarrow \mathbb{R}$ such that for all $f, g \in \mathcal{F}$,

$$
f \succcurlyeq g \Longleftrightarrow I(u \circ f) \geq I(u \circ g),
$$

and such that $I\left(1_{E}\right) \notin\{0,1\}$ for some $E \in \Sigma$. Moreover, $u$ is unique up to a positive affine transformation and $I$ is unique.

It follows from the properties of the functional $I$ that, setting $V(f)=I(u \circ f)$, for every $f \in \mathcal{F}$ and $x \in X$ and dyadic rational $\gamma$,

$$
V(\gamma f \oplus(1-\gamma) x)=\gamma V(f)+(1-\gamma) V(x) .
$$

It can also be shown (Proposition 14 in Appendix B) that the functional $V$ satisfies the separable representation of Lemma 1 for every binary act (rather than just those in $\mathcal{F}_{E}$ ). In fact, let $\rho: \Sigma \rightarrow \mathbb{R}$ be defined by $\rho(A) \equiv I\left(1_{A}\right)$. Then $\rho$ is a capacity and for all $x, y \in X$ such that $x \succcurlyeq y$ and all $A \in \Sigma$,

$$
V(x A y)=u(x) \rho(A)+u(y)(1-\rho(A)) .
$$

Moving beyond binary acts, though, the representation in Theorem 5 allows a great degree of flexibility. Indeed, we show in the next section that both the CEU and MEU models are encompassed by it. Moreover, it is immediate to modify the discussion in Ghirardato and Marinacci [9] to show that most of the decision models which deliver a cardinal state-independent utlity function are special cases of this representation.

The observation of Eq. (11) enables us to address the question of whether the definition of subjective mixture depends on the choice of the essential event $E$. In general, this is possible even if the preference $\succcurlyeq$ satisfies axioms A1-A6. However, such dependence is ruled out when axiom A7 is added (see also Remark 8 below):

Corollary 6 Suppose that $\succcurlyeq$ satisfies axioms $A 1-A 7$ and let $F \neq E$ be an essential event. Then for every $x \succcurlyeq z \succcurlyeq y$,

$$
x E y \sim c_{x E z} E c_{z E y} \quad \text { if and only if } \quad x F y \sim c_{x F z} F c_{z F y} .
$$

That is, for a preference satisfying axioms A1-A7 the definition of $1 / 2: 1 / 2$ (hence dyadic) mixture is independent of the choice of essential event $E$.

Remark 7 In view of the potential dependence of 'subjective mixtures' on the essential event $E$, one could envision a stronger version of axiom $\mathrm{A} 7$ that requires that implication (8) hold for the mixtures defined using any essential event. Corollary 6 shows that all such mixtures agree whenever certainty independence holds for the mixtures defined using $E$. Hence, the alternative formulation of the axiom is not stronger than axiom A7. 
In conclusion, we have shown that under weak preference axioms it is possible to construct a subjective version of the algebraic structure that is assumed in the AA framework. In the next section, we extend three axiomatic models from the AA framework to a fully subjective one, by way of illustrations of the usefulness of this construction.

While the axiomatization that we propose is designed mostly with generality in mind, it is perhaps worth remarking that it is stronger than what is necessary for correctly defining subjective mixtures. In fact, we show in Section 4 that the completeness part of axiom A1 to a large extent can be relaxed without affecting subjective mixtures. The next remark observes that, analogously, the full strength of certainty independence is not needed.

Remark 8 The independence of the notion of even mixture from the essential event $E$ follows from Eq. (11), not from the other properties of the functional $I$. Therefore, the subjective foundation to the AA framework presented here can be extended to the more general 'biseparable preferences' model of Ghirardato and Marinacci [9]. The main benefit of the model introduced here is that the functional $I$ is independent of the specific representation $V$ (which is not necessarily the case for general biseparable preferences). The main cost is the certainty independence property, which is weak and it is satisfied by most models of decision under uncertainty.

\section{Some Immediate Consequences}

It is simple to build on the characterization in Section 2 to provide an axiomatic foundation of some popular models of decision making under uncertainty in a fully subjective environment.

We start with the axiomatization of the classical SEU model of Anscombe and Aumann [1], as well as that of the CEU model of Schmeidler [19]. The key step to obtaining a SEU preference is requiring that axiom A7 hold for every triple of acts:

Axiom A 8 (Weak Independence) For every $f, g, h \in \mathcal{F}$,

$$
f \sim g \Longrightarrow \frac{1}{2} f \oplus \frac{1}{2} h \sim \frac{1}{2} g \oplus \frac{1}{2} h
$$

On the other hand, CEU follows if we weaken axiom A8 by requiring that the implication holds only for acts which are 'commonly monotonic', in the following sense: $f, g \in \mathcal{F}$ are comonotonic if there are no $s, s^{\prime} \in S$ such that $f(s) \succ f\left(s^{\prime}\right)$ and $g\left(s^{\prime}\right) \succ g(s)$.

Axiom A 9 (Weak Comonotonic Independence) For every $f, g, h \in \mathcal{F}$, such that $f, g$ and $h$ are pairwise comonotonic,

$$
f \sim g \Longrightarrow \frac{1}{2} f \oplus \frac{1}{2} h \sim \frac{1}{2} g \oplus \frac{1}{2} h .
$$


Notice that, differently from axiom A7, both these axioms require that the implications hold only for $\gamma=(1 / 2)$.

In either case, the functional $I$ of Theorem 5 becomes a Choquet integral with respect to a function that represents the DM's beliefs. ${ }^{9}$ Depending on whether independence holds only for comonotonic acts or for all acts, such function is either a capacity or a probability.

Proposition 9 Let $\succcurlyeq$ be a binary relation on $\mathcal{F}$. Then $\succcurlyeq$ satisfies axioms $A 1-A 6$ and A8 (resp. A9) if and only if there exist a $\tau$-continuous nonconstant function $u: X \rightarrow \mathbb{R}$ and a probability (resp. capacity) $P$ on $\Sigma$ such that the functional $I: B(\Sigma) \rightarrow \mathbb{R}$ defined by

$$
I(\varphi)=\int_{S} \varphi d P
$$

represents $\succcurlyeq$ in the sense of $E q$. (9), and such that $P(E) \in(0,1)$. Moreover, $u$ is unique up to a positive affine transformation and $P$ is unique.

Next, we offer a subjective axiomatization of Gilboa and Schmeidler's maxmin expected utility model [11]. Here it is key to add the following axiom, which is analogous (but slightly weaker) to the axiom Gilboa and Schmeidler call 'uncertainty aversion'. ${ }^{10}$

Axiom A 10 (Ambiguity Hedging) For every $f, g \in \mathcal{F}$,

$$
f \sim g \Longrightarrow \frac{1}{2} f \oplus \frac{1}{2} g \succcurlyeq f \text {. }
$$

Intuitively, a DM displays ambiguity hedging if he prefers the 'even mixture' of indifferent acts (which possibly hedges ambiguity) to either of the 'pure' acts (which certaintly do not). A symmetric axiom, called 'ambiguity speculating', describes a DM with opposite preferences.

It is worth underscoring that, since the mixtures used in the axiom are subjective, ambiguity hedging does not describe, as its counterpart in the AA framework, a preference for 'objective' randomization. Rather, it embodies a preference for utility smoothing, which is completely unrelated to the decision maker's attitude towards possible randomized choices. The latter interpretation makes, at least in our opinion, ambiguity hedging a much more palatable property than its AA analogue.

Adding axiom A10 to those in the previous section allows a full description of the functional $I$ in Theorem 5. (If we used 'ambiguity speculating' instead, we would get a 'max' rather than a 'min'.)

\footnotetext{
9 See Appendix A for a definition, and notice that a Choquet integral with respect to a probability is a standard integral.

10 See Ghirardato and Marinacci [8] for a discussion of such terminology, motivating our departure from it.
} 
Proposition 10 Let $\succcurlyeq$ be a binary relation on $\mathcal{F}$. Then $\succcurlyeq$ satisfies axioms A1-A7 and $A 10$ if and only if there exist a $\tau$-continuous nonconstant function $u: X \rightarrow \mathbb{R}$ and a nonempty, closed and convex set $C$ of probabilities on $\Sigma$ such that the functional $I: B(\Sigma) \rightarrow \mathbb{R}$ defined by

$$
I(\varphi)=\min _{P \in C} \int_{S} \varphi d P,
$$

represents $\succcurlyeq$ in the sense of $E q .(9)$, and such that $\left[\min _{P \in C} P(E), \max _{P \in C} P(E)\right] \subseteq(0,1)$. Moreover, $u$ is unique up to a positive affine transformation and $C$ is unique.

\section{A Subjective Axiomatization of Bewley's Model}

In this final section, we modify the model of Section 2 to provide a subjective foundation to Bewley's [2] model of choice with incomplete preferences.

Besides showing that the full strength of completeness is not necessary for defining subjective mixtures, the exercise serves the purpose of demonstrating that subjective mixtures can be helpful in modelling choice also when ambiguity aversion is not a motivating factor. In fact, incompleteness of preferences may plausibly be due to causes unrelated to ambiguity. Moreover, the model we present here does not embody ambiguity aversion, as it remains agnostic on the procedure by which the DM resolves incomparabilities (one possible solution is Bewley's 'inertia' assumption, which does not reflect ambiguity aversion).

For this section only, we add further structure to the set of consequences $X$. We also assume, as Bewley [2] did, that $S$ is finite.

Structural Assumption The set $X$ is a connected, separable and compact topological space with topology $\tau$. The set $S$ is finite.

As in Section 2, our axiomatization comprises seven axioms, labelled B1-B7. Axioms $\mathrm{B} 2, \mathrm{~B} 4$ and $\mathrm{B} 6$ are exactly axioms A2, A4 and A6. Therefore, we do not restate them. We only state the axioms which, though analogous to their 'A' counterpart, need adaptation to the present setting. The first two axioms parallel axioms A1 and A3, but here we require that $\succcurlyeq$ is complete only on the set of the acts measurable with respect to the essential event $E$.

Axiom B 1 (Preorder) (a) For all $f \in \mathcal{F}, f \succcurlyeq f$. (b) For all $f, g, h \in \mathcal{F}$, if $f \succcurlyeq g$ and $g \succcurlyeq h$, then $f \succcurlyeq h$.

Axiom B 3 (Complete Essentiality) There exists an essential event $E \in \Sigma$ such that $\succcurlyeq$ is complete on $\mathcal{F}_{E}$. 
The next axiom imposes a stronger continuity requirement than axiom A5, needed in order to compensate the incompleteness of $\succcurlyeq$.

Axiom B 5 (Continuity) Let $\left\{f_{\alpha}\right\}_{\alpha \in D} \subseteq \mathcal{F}$ be a net that pointwise converges to $f \in \mathcal{F}$ and $\left\{g_{\alpha}\right\}_{\alpha \in D} \subseteq \mathcal{F}$ be a net that pointwise converges to $g \in \mathcal{F}$. If $f_{\alpha} \succcurlyeq g_{\alpha}$ for all $\alpha \in D$, then $f \succcurlyeq g$.

As before, it is possible to show that if $\succcurlyeq$ satisfies axioms B1, B2, B3 and B5, every act $f \in \mathcal{F}_{E}$ has a certainty equivalent $c_{f}$ (notice that this need not be true of acts outside $\left.\mathcal{F}_{E}\right)$.

As all acts involved in the definition of $(1 / 2) x \oplus(1 / 2) y$ belong to $\mathcal{F}_{E}$, the definition of mixture is unchanged from the previous section. We can thus state the independence axiom, which is stronger than the previous versions, as it applies to every triple of acts.

Axiom B 7 (Independence) For every $f, g, h \in \mathcal{F}$ and every dyadic rational $\gamma$,

$$
f \succcurlyeq g \Longrightarrow \gamma f \oplus(1-\gamma) h \succcurlyeq \gamma g \oplus(1-\gamma) h .
$$

Our last result shows that, unsurprisingly, axioms B1-B7 yield a representation of preferences weaker than Proposition 9: a DM satisfying the axioms has a family of probabilities as beliefs, and prefers act $f$ to act $g$ when the expected utility of $f$ is unanimously greater than the expected utility of $g$.

Theorem 11 Let $\succcurlyeq$ be a binary relation on $\mathcal{F}$. Then $\succcurlyeq$ satisfies axioms B1-B 7 if and only if there exist a $\tau$-continuous nonconstant function $u: X \rightarrow \mathbb{R}$ and a nonempty, closed and convex set $C$ of probabilities on $\Sigma$ such that for all $f, g \in \mathcal{F}$,

$$
f \succcurlyeq g \Longleftrightarrow \int_{S}(u \circ f) d P \geq \int_{S}(u \circ g) d P \quad \text { for all } P \in C,
$$

and such that $P(E)=P^{\prime}(E) \in(0,1)$ for all $P, P^{\prime} \in C$ for some $E \in \Sigma$. Moreover, $u$ is unique up to a positive affine transformation and $C$ is unique.

It is simple to see that a result analogous to Corollary 6 holds in this setting: If $F \neq E$ is an essential event such that $\succcurlyeq$ is complete on $\mathcal{F}_{F}$, then $x F y \sim c_{x F z} F c_{z F y}$ if and only if $x E y \sim c_{x E z} F c_{z E y}$. 


\section{Appendix A An Integral for Capacities}

The notion of integral used for capacities is the Choquet integral: For a given $\Sigma$ measurable function $\varphi: S \rightarrow \mathbb{R}$, the Choquet integral of $\varphi$ with respect to a capacity $\nu$ is defined as:

$$
\int_{S} \varphi d \nu=\int_{0}^{\infty} \nu(\{s \in S: \varphi(s) \geq \alpha\}) d \alpha+\int_{-\infty}^{0}[1-\nu(\{s \in S: \varphi(s) \geq \alpha\})] d \alpha
$$

where the r.h.s. is a Riemann integral (which is well defined because $\nu$ is monotone). When $\nu$ is additive, (12) becomes a standard (additive) integral. In general it is seen to be monotonic, positive homogeneous and comonotonic additive: If $\varphi, \psi: S \rightarrow \mathbb{R}$ are

comonotonic, then $\int(\varphi+\psi) d \nu=\int \varphi d \nu+\int \psi d \nu$. Two functions $\varphi, \psi: S \rightarrow \mathbb{R}$ are called comonotonic if there are no $s, s^{\prime} \in S$ such that $\varphi(s)>\varphi\left(s^{\prime}\right)$ and $\psi(s)<\psi\left(s^{\prime}\right)$.

\section{Appendix B Proofs}

\section{B.1 Proof of Lemma 1}

We show that axioms A1-A6 imply that axioms 1-6 of Nakamura [16] hold on $\mathcal{F}_{E}$. Axiom A2 immediately implies that axioms 1 and 4 are satisfied. The fact that our axiom A4 is equivalent to his 3 follows from these simple observations: an event $A$ is 'null' (resp. 'universal') in the sense of Nakamura if and only if $A^{c}$ is universal (resp. null), while if $A$ is essential, then $A^{c} \in \Sigma_{E}$ is neither 'null' nor 'universal' in his sense. Axiom 6 is clearly equivalent to our axiom A6.

To show that his axioms 2 and 5 hold, we use the following lemma. As it is analogous to Lemma 29 in [9], we omit its proof.

Lemma 12 Let $\succcurlyeq$ be a binary relation satisfying axioms A1, A2, A3 and A5. If $X$ is connected, then

(a) for every $f \in \mathcal{F}$ there exists $x \in X$ such that $f \sim x$.

(b) for every $x, y, z \in X, A \in \Sigma_{E}$, and $f \in \mathcal{F}$, if $x A z \succ f \succ y A z$, there exists $x^{\prime} \in X$ such that $x^{\prime} A z \sim f$.

Part (b) is exactly Nakamura's axiom 2. As to axiom 5, it is possible to mimic the proof of Lemma 30 of [9] to show that Lemma 12 and axiom A4 imply its validity.

It now follows from Theorem 1 of Nakamura [16] that there exists a unique $\rho_{E}$ on $\Sigma_{E}$ and a function $u: X \rightarrow \mathbb{R}$, unique up to positive affine transformations, such that the functional $V: \mathcal{F}_{E} \rightarrow \mathbb{R}$ defined by Eq. (6) represents $\succcurlyeq$ on $\mathcal{F}_{E}$. We extend $V$ to all of $\mathcal{F}$ by letting $V(f)=u\left(c_{f}\right)$ for any $f \in \mathcal{F}$. It is immediate to check that, thus defined, 
$V$ represents $\succcurlyeq$. Moreover, $u$ is shown to be $\tau$-continuous (see the proof of Lemma 31 in $[9])$.

The necessity of axioms A1-A2 is obvious, and that of axiom A6 is proved in $[16$, Theorem 1]. As to axiom A3, it suffices to observe that since $\rho_{E}(E) \in(0,1)$ and $u$ is nonconstant, we have

$$
u(x)>u(x) \rho_{E}(E)+u(y)\left(1-\rho_{E}(E)\right)>u(y)
$$

for some $x \succ y$, proving that $E$ is essential. Axiom A4 similarly follows from the observation that $A \in \Sigma_{E}$ is null (resp. universal) iff $\rho_{E}(A)=0$ (resp. $\rho_{E}(A)=1$ ). To show that axiom A5 holds, assume that $f_{\alpha}=x_{\alpha} E y_{\alpha} \rightarrow x E y=f$ pointwise and that $f_{\alpha} \succcurlyeq g$. Since $u$ is $\tau$-continuous, we have that

$$
\max \left\{\left|u\left(x_{\alpha}\right)-u(x)\right|,\left|u\left(y_{\alpha}\right)-u(y)\right|\right\} \rightarrow 0 .
$$

Consequently, $u \circ\left(x_{\alpha} E y_{\alpha}\right) \rightarrow u \circ(x E y)$ uniformly, whence

$$
V\left(f_{\alpha}\right)=\int\left[u \circ\left(x_{\alpha} E y_{\alpha}\right)\right] d \rho_{E} \rightarrow \int[u \circ(x E y)] d \rho_{E}=V(f)
$$

where the integrals are taken in the sense of Choquet [6]. This shows that $f \succcurlyeq g$, and concludes the proof of necessity.

The uniqueness of $V$ is shown as follows: Suppose that $V^{\prime}: \mathcal{F} \rightarrow \mathbb{R}$ is another functional satisfying the representation, which corresponds to a utility $u^{\prime}$ and capacity $\rho_{E}^{\prime}$. By the uniqueness properties mentioned above, $u^{\prime}=a u+b$ for $a>0$ and $b \in \mathbb{R}$ and $\rho_{E}^{\prime}=\rho_{E}$. Therefore

$$
V^{\prime}(f)=u^{\prime}\left(c_{f}\right)=a u\left(c_{f}\right)+b=a V(f)+b .
$$

\section{B.2 Proof of Lemma 3}

Assume w.l.o.g. that $x \succcurlyeq y$, and suppose that there is a $z$ satisfying the conditions of Definition 2. By axiom A2 and the fact that $x \succcurlyeq z \succcurlyeq y$, it follows that $x E z \succcurlyeq z E y$. Let $r=\rho_{E}(E) \in(0,1)$. Applying the representation of Lemma 1, we have

$$
\begin{aligned}
& V(x E z)=u(x) r+u(z)(1-r) \\
& V(z E y)=u(z) r+u(y)(1-r) .
\end{aligned}
$$

Using these equations, we obtain

$$
\begin{aligned}
V\left(c_{x E z} E c_{z E y}\right) & =u\left(c_{x E z}\right) r+u\left(c_{z E y}\right)(1-r) \\
& =V(x E z) r+V(z E y)(1-r) \\
& =[u(x) r+u(z)(1-r)] r+[u(z) r+u(y)(1-r)](1-r) \\
& =u(x) r^{2}+u(y)(1-r)^{2}+2 u(z) r(1-r),
\end{aligned}
$$


and

$$
\begin{aligned}
V(x E y) & =u(x) r+u(y)(1-r) \\
& =u(x) r^{2}+u(y)(1-r)^{2}+[u(x)+u(y)] r(1-r) .
\end{aligned}
$$

Thus, $V(x E y)=V\left(c_{x E z} E c_{z E y}\right)$ is equivalent to

$$
\frac{u(x)+u(y)}{2}=u(z) .
$$

By the continuity of $u$ and the connectedness of $X$, for all $x \succcurlyeq y$ there exists a $z \in X$ such that Eq. (13) is satisfied. By the above argument, such $z=(1 / 2) x \oplus(1 / 2) y$.

\section{B.3 Proof of Theorem 5}

Let $u$ and $V$ be respectively the utility and functional from Lemma 1 . We start with a simple observation on dyadic mixtures:

Lemma 13 Let $x, y \in X$ and $\gamma$ be a dyadic rational. Then

$$
u(\gamma x \oplus(1-\gamma) y)=\gamma u(x)+(1-\gamma) u(y)
$$

Proof: If $\gamma=\sum_{i=1}^{\infty} a_{i} / 2^{i}$, define $\ell(\gamma)=\max \left\{i \geq 1: a_{i}=1\right\}$. The proof is by induction on $\ell(\gamma)$. If $\ell(\gamma)=1$, then $\gamma=1 / 2$ and the result follows from Lemma 3 .

Suppose that the result holds for all $\gamma$ such that $\ell(\gamma) \leq n$, and let $\gamma=\sum_{i=1}^{n+1} a_{i} / 2^{i}$ (where $a_{n+1}=1$, otherwise the result is true by the induction hypothesis). Let $\delta=$ $\sum_{i=1}^{n} a_{i+1} / 2^{i}$, so that $\gamma=(1 / 2) a_{1}+(1 / 2) \delta$. Then,

$$
\begin{aligned}
u(\gamma x \oplus(1-\gamma) y) & =u\left(\frac{1}{2} z_{1} \oplus \frac{1}{2}(\delta x \oplus(1-\delta) y)\right) \\
& =\frac{1}{2} u\left(z_{1}\right)+\frac{1}{2}(\delta u(x)+(1-\delta) u(y))
\end{aligned}
$$

If $a_{1}=0\left(\right.$ resp. $\left.a_{1}=1\right)$, then $z_{1}=y\left(\right.$ resp. $\left.z_{1}=x\right)$ and the above equation is rewritten as

$$
\begin{aligned}
u(\gamma x \oplus(1-\gamma) y) & =\frac{1}{2} \delta u(x)+\left(1-\frac{1}{2} \delta\right) u(y) \quad\left(\text { resp. }=\left(\frac{1}{2}+\frac{1}{2} \delta\right) u(x)+\frac{1}{2}(1-\delta) u(y)\right) \\
& =\gamma u(x)+(1-\gamma) u(y) .
\end{aligned}
$$

This concludes the proof.

Since $u$ is nonconstant, we can choose $u$ s.t. $u(X) \supseteq[-1,1]$. Denote by $B(\Sigma, u(X))$ the subset of $B(\Sigma)$ consisting of simple measurable functions with range in $u(X)$. It is simple to show that $B(\Sigma, u(X))=\{u \circ f: f \in \mathcal{F}\}$. 
Define $I^{\prime}(u \circ f)=V(f)$ for all $f \in \mathcal{F}$. Clearly, $u \circ f=u \circ g$ iff $u(f(s))=u(g(s))$ for all $s \in S$ iff $f(s) \sim g(s)$ for all $s \in S$ only if $V(f)=V(g)$ (by axiom A2). Hence, $I^{\prime}: B(\Sigma, u(X)) \rightarrow \mathbb{R}$ is well defined. It is also monotonic: If $\varphi=u \circ f$ and $\psi=u \circ g$ and $\varphi \geq \psi$, then $u(f(s)) \geq u(g(s))$ for all $s \in S$, which by axiom A2 implies $f \succcurlyeq g$ so that $I^{\prime}(\varphi)=I^{\prime}(u \circ f)=V(f) \geq V(g)=I^{\prime}(u \circ g)=I^{\prime}(\psi)$. Finally, for every $b=u(x)$ for $x \in X, I^{\prime}(b)=I^{\prime}(u(x))=V(x)=u(x)=b$.

Take a dyadic rational $\gamma$, a $\varphi=u \circ f \in B(\Sigma, u(X))$ and $b \in u(X)$. Denote by $x$ an element in $X$ such that $u(x)=b$. By Lemma 13,

$$
u \circ(\gamma f \oplus(1-\gamma) x)=\gamma u \circ f+(1-\gamma) u(x)=\gamma \varphi+(1-\gamma) b
$$

By axiom A7, $f \sim c_{f}$ implies $\gamma f \oplus(1-\gamma) x \sim \gamma c_{f} \oplus(1-\gamma) x$. We thus obtain

$$
\begin{aligned}
I^{\prime}(\gamma \varphi+(1-\gamma) b) & =V(\gamma f \oplus(1-\gamma) x) \\
& =u\left(\gamma c_{f} \oplus(1-\gamma) x\right) \\
& =\gamma V(f)+(1-\gamma) u(x) \\
& =\gamma I^{\prime}(\varphi)+(1-\gamma) b .
\end{aligned}
$$

In particular, setting $b=0$ in the equation above yields $I^{\prime}(\gamma \varphi)=\gamma I^{\prime}(\varphi)$.

We now extend $I^{\prime}$ to a functional $I: B(\Sigma) \rightarrow \mathbb{R}$ as follows:

$$
I(\psi)=2^{n} I^{\prime}\left(\frac{1}{2^{n}} \psi\right) \quad \text { if } \quad \frac{1}{2^{n}} \psi \in B(\Sigma, u(X)) .
$$

$I$ is well defined: if $\left(1 / 2^{m}\right) \psi \in B(\Sigma, u(X))$ for, say, $m=n+p$, then, using the facts that $1 / 2^{p}$ is dyadic and that $\left(1 / 2^{n}\right) \psi \in B(\Sigma, u(X))$,

$$
2^{m} I\left(\frac{1}{2^{m}} \psi\right)=2^{n} 2^{p} I\left(\frac{1}{2^{p}} \frac{1}{2^{n}} \psi\right)=2^{n} I\left(\frac{1}{2^{n}} \psi\right) .
$$

We now show that $I(\gamma \varphi+(1-\gamma) b)=\gamma I(\varphi)+(1-\gamma) b$ for every dyadic rational $\gamma$, every $\varphi \in B(\Sigma)$ and every $b \in \mathbb{R}$. Let $q \in \mathbb{N}$ be such that $\varphi / 2^{q} \in B(\Sigma, u(X))$ and $b / 2^{q} \in u(X)$. Then

$$
\begin{aligned}
I(\gamma \varphi+(1-\gamma) b) & =2^{q} I^{\prime}\left(\gamma \varphi / 2^{q}+(1-\gamma) b / 2^{q}\right) \\
& =2^{q}\left[\gamma I^{\prime}\left(\varphi / 2^{q}\right)+(1-\gamma) b / 2^{q}\right] \\
& =\gamma 2^{q} I^{\prime}\left(\varphi / 2^{q}\right)+(1-\gamma) b \\
& =\gamma I(\varphi)+(1-\gamma) b .
\end{aligned}
$$

As a consequence of this equality, $I(\varphi / 2)=I(\varphi) / 2$ for every $\varphi \in B(\Sigma)$. Using this result and the equality again yields $I(\varphi+b)=I(\varphi)+b$. Therefore $I$ is c-additive.

The monotonicity of $I$ descends from the monotonicity of $I^{\prime}$. Sup-norm continuity of $I$ then follows from monotonicity and c-additivity. The positive homogeneity of $I$ then 
follows from the property that $I(\gamma \varphi)=\gamma I(\varphi)$ for every dyadic rational $\gamma$ and sup-norm continuity. We thus conclude that $I$ is c-linear, proving sufficiency.

To prove necessity, we show that the representation $V$ has a special form on all binary acts.

Proposition 14 Let $\succcurlyeq$ be the binary relation on $\mathcal{F}$ induced by the functional $V$ defined by $V(f)=I(u(f))$, where $I: B(\Sigma) \rightarrow \mathbb{R}$ is a monotonic c-linear functional and $u: X \rightarrow \mathbb{R}$ is a nonconstant function. Then there exists a unique capacity $\rho: \Sigma \rightarrow[0,1]$ such that for every $x \succcurlyeq y$ and $A \in \Sigma$,

$$
V(x A y)=u(x) \rho(A)+u(y)(1-\rho(A)) .
$$

Proof: Set $\rho(A)=I\left(1_{A}\right)$ for all $A \in \Sigma$. Consider $x \succcurlyeq y$ and $A \in \Sigma$. Then

$$
\begin{aligned}
V(x A y) & =I[u \circ(x A y)] \\
& =I\left[(u(x)-u(y)) 1_{A}+u(y)\right] \\
& =u(x) \rho(A)+u(y)(1-\rho(A)) .
\end{aligned}
$$

To show uniqueness, assume that $\rho^{\prime}$ also satisfies Eq. (14) and choose $x \succ y$. We have

$$
\begin{aligned}
\rho^{\prime}(A) & =\frac{V(x A y)-u(y)}{u(x)-u(y)} \\
& =\frac{I\left[(u(x)-u(y)) 1_{A}+u(y)\right]-u(y)}{u(x)-u(y)} \\
& =\frac{u(x)-u(y)}{u(x)-u(y)} I\left(1_{A}\right),
\end{aligned}
$$

which concludes the proof.

Since the proposition shows that the utility $u$ and capacity $\rho$ are such that $V$ satisfies Eq. (6), it now follows from Lemma 1 that axioms A1-A6 must hold. Axiom A7 is clearly necessary.

We close with the proof of the uniqueness of $I$ and cardinality of $u$. By the proposition and Lemma 1, $V$ and $u$ are cardinal and $\rho_{E}=\rho$. Suppose that $I^{\prime}: B(\Sigma) \rightarrow \mathbb{R}$ and $u^{\prime}$ correspond to another representation of $\succcurlyeq$. Then, $V^{\prime}=I^{\prime} \circ u^{\prime}=a V+b$ with $a>0$ and $b \in \mathbb{R}$ and for all $f \in \mathcal{F}$

$$
a I(u \circ f)+b=I^{\prime}\left(u^{\prime} \circ f\right)=I^{\prime}(a(u \circ f)+b)=a I^{\prime}(u \circ f)+b .
$$

This shows that $I^{\prime}=I$ on $B(\Sigma, u(X))$. By positive homogeneity, $I^{\prime} \equiv I$. 


\section{B.4 Proof of Proposition 9}

We prove the statement for the case of a preference satisfying axioms A1-A6 and A9. The other case is proved analogously (just remove all references to 'comonotonic' and change 'capacity' into 'probability').

As in the proof of Theorem 5, we consider the utility $u$ and functional $V$ from Lemma 1 , chosen so that $u(X) \supseteq[-1,1]$. Observe that then $f, g \in \mathcal{F}$ are comonotonic iff $u \circ f$ and $u \circ g$ are comonotonic. We next define $I^{\prime}(u \circ f)=V(f)$ for all $f \in \mathcal{F}$. As before, $I^{\prime}$ is well-defined and monotonic, and $I^{\prime}(b)=b$. Given comonotonic $\varphi, \psi \in B(\Sigma, u(X))$, let $\varphi=u \circ f$ and $\psi=u \circ g$. By Lemma 3,

$$
u \circ((1 / 2) f \oplus(1 / 2) g)=(1 / 2) u \circ f+(1 / 2) u \circ g=(1 / 2) \varphi+(1 / 2) \psi .
$$

By axiom A9, $f \sim c_{f}$ and $g \sim c_{g}$ imply

$$
(1 / 2) f \oplus(1 / 2) g \sim(1 / 2) c_{f} \oplus(1 / 2) g \sim(1 / 2) c_{f} \oplus(1 / 2) c_{g} .
$$

We thus obtain

$$
\begin{aligned}
I^{\prime}((1 / 2) \varphi+(1 / 2) \psi) & =V((1 / 2) f \oplus(1 / 2) g) \\
& =u\left((1 / 2) c_{f} \oplus(1 / 2) c_{g}\right) \\
& =(1 / 2) V(f)+(1 / 2) V(g) \\
& =(1 / 2) I^{\prime}(\varphi)+(1 / 2) I^{\prime}(\psi) .
\end{aligned}
$$

This implies $I^{\prime}((1 / 2) \varphi)=(1 / 2) I^{\prime}(\varphi)$, so that $I^{\prime}\left((1 / 2)^{n} \varphi\right)=(1 / 2)^{n} I^{\prime}(\varphi)$ for all $n \in \mathbb{N}$.

We now extend $I^{\prime}$ to a functional $I: B(\Sigma) \rightarrow \mathbb{R}$ as follows:

$$
I(\psi)=2^{n} I^{\prime}\left(\frac{1}{2^{n}} \psi\right) \quad \text { if } \quad \frac{1}{2^{n}} \psi \in B(\Sigma, u(X)) .
$$

Again, $I$ is well defined. We now show that $I((1 / 2) \varphi+(1 / 2) \psi)=(1 / 2) I(\varphi)+(1 / 2) I(\psi)$, for every comonotonic $\varphi, \psi \in B(\Sigma)$. Let $q \in \mathbb{N}$ be such that $\varphi / 2^{q}, \psi / 2^{q} \in B(\Sigma, u(X))$. Then

$$
\begin{aligned}
I((1 / 2) \varphi+(1 / 2) \psi) & =2^{q} I^{\prime}\left((1 / 2) \varphi / 2^{q}+(1 / 2) \psi / 2^{q}\right) \\
& =2^{q}\left[(1 / 2) I^{\prime}\left(\varphi / 2^{q}\right)+(1 / 2) I^{\prime}\left(\psi / 2^{q}\right)\right] \\
& =(1 / 2) 2^{q} I^{\prime}\left(\varphi / 2^{q}\right)+(1 / 2) 2^{q} I^{\prime}\left(\psi / 2^{q}\right) \\
& =(1 / 2) I(\varphi)+(1 / 2) I(\psi) .
\end{aligned}
$$

As a consequence of this equality, $I(\varphi / 2)=I(\varphi) / 2$ for every $\varphi \in B(\Sigma)$. Using this result and the equality again yields $I(\varphi+\psi)=I(\varphi)+I(\psi)$. That is, $I$ is comonotonic additive.

Since $I$ is comonotonic additive, monotonic (the monotonicity of $I$ descends from the monotonicity of $I^{\prime}$ ) and satisfies $I(b)=b$ for all $b \in \mathbb{R}$, there exists a unique capacity $P$ on $\Sigma$ such that $I(\varphi)=\int \varphi d P$, where the integral is taken in the sense of Choquet. This proves sufficiency. Necessity and uniqueness are straightforward. 


\section{B.5 Proof of Proposition 10}

Consider the function $u$ and the monotonic c-linear functional $I$ in Theorem 5 . We now show that, if we add axiom A10, the functional $I$ is also superadditive. This is shown to yield the desired representation (see, e.g., Marinacci [15]).

For all $\varphi=u \circ f$ and $\psi=u \circ g$ such that $I(\varphi)=I(\psi)$, we have

$$
I\left(\frac{1}{2} \varphi+\frac{1}{2} \psi\right)=I\left(u \circ\left(\frac{1}{2} f \oplus \frac{1}{2} g\right)\right) \geq I(u \circ f)=I(\varphi)=\frac{1}{2} I(\varphi)+\frac{1}{2} I(\psi) .
$$

Given $\varphi, \psi \in B(\Sigma)$, suppose first that $I(\varphi)=I(\psi)$. Choose $\alpha \in(0,1]$ such that $\alpha \varphi, \alpha \psi \in$ $B(\Sigma, u(X))$. By the previous observation

$$
I\left(\frac{1}{2} \alpha \varphi+\frac{1}{2} \alpha \psi\right) \geq \frac{1}{2} I(\alpha \varphi)+\frac{1}{2} I(\alpha \psi)
$$

which by homogeneity of $I$ implies $I(\varphi+\psi) \geq I(\varphi)+I(\psi)$. If, on the other hand, $I(\varphi) \neq I(\psi)$, let $\gamma=I(\varphi)-I(\psi)$ and apply c-linearity and the previous argument to $\varphi$ and $\psi+\gamma$. This proves the superadditivity of $I$.

\section{B.6 Proof of Theorem 11}

Necessity is straightforward. We prove sufficiency. We depart from the observation that, as we did in Section 2, we can use axioms B1-B6 to show that the preference $\succcurlyeq$ has a separable representation on $\mathcal{F}_{E}$. This time, however, the representation is not extendable to all $\mathcal{F}$.

Lemma $15 \succcurlyeq i s$ a binary relation satisfying axioms B1-B6 if and only if there is a $\tau$ continuous nonconstant utility index $u: X \rightarrow \mathbb{R}$ and a capacity $\rho_{E}: \Sigma_{E} \rightarrow[0,1]$, with $\rho_{E}(E) \in(0,1)$, such that the functional $V: \mathcal{F}_{E} \rightarrow \mathbb{R}$ defined by $V(f) \equiv u\left(c_{f}\right)$ for any $f \in \mathcal{F}_{E}$ represents $\succcurlyeq$, it is monotonic and it satisfies, for all $x \succcurlyeq y$ and all $A \in \Sigma_{E}$,

$$
V(x A y)=u(x) \rho_{E}(A)+u(y)\left(1-\rho_{E}(A)\right) .
$$

Moreover, such $u$ and $V$ are unique up to a positive affine transformation and $\rho_{E}$ is unique.

Proof: This is analogous to the proof of Lemma 1. The major difference is that only the acts in $\mathcal{F}_{E}$ necessarily have certainty equivalents.

Let $u$ and $V$ be respectively the utility and functional from the Lemma. Since $u$ is nonconstant, we can choose $u$ s.t. $u(X) \supseteq[-1,1]$. We define a binary relation on $B(\Sigma, u(X))$ as follows: For all $f, g \in \mathcal{F}$, we let $u \circ f \succsim_{1} u \circ g$ iff $f \succcurlyeq g$. The following are then true: 
- $\succsim_{1}$ is well-defined and monotonic: Assume $u \circ f=u \circ f^{\prime}$ and $u \circ g=u \circ g^{\prime}$. By monotonicity $f \sim f^{\prime}$ and $g \sim g^{\prime}$. Then $f \succcurlyeq g$ iff $f^{\prime} \succcurlyeq g^{\prime}$. The proof of monotonicity is obvious.

- $\succsim_{1}$ is reflexive: for all $f \in \mathcal{F}, f \sim f$, hence $u \circ f \sim_{1} u \circ f$.

- $\succsim_{1}$ is transitive: If $u \circ f \succsim_{1} u \circ g$ and $u \circ g \succsim_{1} u \circ h$, then $f \succcurlyeq g \succcurlyeq h$, hence $f \succcurlyeq h$ implying $u \circ f \succsim_{1} u \circ h$.

- $\succsim_{1}$ is continuous: Assume that $u \circ f_{\alpha} \rightarrow u \circ f$ and $u \circ g_{\alpha} \rightarrow u \circ g$ and $u \circ f_{\alpha} \succsim_{1} u \circ g_{\alpha}$, implying $f_{\alpha} \succcurlyeq g_{\alpha}$, for all $\alpha \in D$. Taking subnets we can assume that $f_{\beta} \rightarrow f^{\prime}$ and $g_{\beta} \rightarrow g^{\prime}$ (as $\mathcal{F}=X^{S}$ is compact). By axiom B5 $f^{\prime} \succcurlyeq g^{\prime}$, whence $u \circ f^{\prime} \succsim_{1} u \circ g^{\prime}$. Finally, we observe that $u \circ f^{\prime}=u \circ f$ and $u \circ g^{\prime}=u \circ g$ since $u \circ f_{\beta} \rightarrow u \circ f$ and $u \circ g_{\beta} \rightarrow u \circ g$. This shows that $u \circ f \succsim_{1} u \circ g$.

- $\succsim_{1}$ is independent (with $\gamma \in[0,1]$ ): Let $f, g, h \in \mathcal{F}$ and suppose that $u \circ f \succsim_{1} u \circ g$. Then $f \succcurlyeq g$ implying $\gamma f \oplus(1-\gamma) h \succcurlyeq \gamma g \oplus(1-\gamma) h$ for every dyadic rational $\gamma$ by axiom B7. This implies, since clearly Lemma 13 extends to this case,

$$
\begin{aligned}
\gamma u \circ f+(1-\gamma) u \circ h & =u \circ(\gamma f \oplus(1-\gamma) h) \\
& \succsim_{1} u \circ(\gamma g \oplus(1-\gamma) h) \\
& =\gamma u \circ g+(1-\gamma) u \circ h .
\end{aligned}
$$

By continuity, independence holds for every $\gamma \in[0,1]$.

Notice that, by the independence and continuity of $\succsim_{1}$, it follows that for all $\varphi, \psi \in$ $B(\Sigma, u(X))$ and all $\gamma \in(0,1]: \varphi \succsim_{1} \psi$ iff $\gamma \varphi \succsim_{1} \gamma \psi$ (the proof is similar to that of Lemma 1.2 in Shapley and Baucells [20]).

We next define a binary relation $\succsim_{2}$ on $B(\Sigma)$ as follows: For all $\varphi, \psi \in B(\Sigma), \varphi \succsim_{2} \psi$ iff $\gamma \varphi \succsim_{1} \gamma \psi$ for some $\gamma \in(0,1]$.

- $\succsim_{2}$ is well-defined and monotonic: Let $\varphi, \psi \in B(\Sigma)$ and $\alpha, \beta \in(0,1]$ be such that $\alpha \varphi, \beta \varphi, \alpha \psi, \beta \psi \in B(\Sigma, u(X))$. W.l.o.g. $\beta=\gamma \alpha$ with $\gamma \in(0,1]$. It follows from the observation above that $\alpha \varphi \succsim_{1} \alpha \psi$ iff $\gamma \alpha \varphi \succsim_{1} \gamma \alpha \psi$ iff $\beta \varphi \succsim_{1} \beta \psi$. Monotonicity is trivial.

- $\succsim_{2}$ is obviously reflexive and transitive (i.e., a preorder). It is also nontrivial.

- $\succsim_{2}$ is independent and positively homogenous: For $\varphi, \psi, \xi \in B(\Sigma)$ suppose that $\varphi \succsim_{2} \psi$ and consider $\alpha \in(0,1]$ such that $\alpha \varphi, \alpha \psi, \alpha \xi \in B(\Sigma, u(X))$. Then $\alpha \varphi \succsim_{1} \alpha \psi$ and by independence of $\succsim_{1}$ for every $\gamma \in[0,1]$,

$$
\begin{aligned}
\gamma \alpha \varphi+(1-\gamma) \alpha \xi \succsim_{1} \gamma \alpha \psi+(1-\gamma) \alpha \xi & \Leftrightarrow \alpha(\gamma \varphi+(1-\gamma) \xi) \succsim_{1} \alpha(\gamma \psi+(1-\gamma) \xi) \\
& \Leftrightarrow \gamma \varphi+(1-\gamma) \xi \succsim_{2} \gamma \psi+(1-\gamma) \xi .
\end{aligned}
$$

The proof of positive homogeneity is now immediate. 
- $\succsim_{2}$ is continuous: Suppose that $\varphi_{n}, \varphi, \psi_{n}, \psi \in B(\Sigma), \varphi_{n} \rightarrow \varphi, \psi_{n} \rightarrow \psi$ and $\varphi_{n} \succsim_{2}$ $\psi_{n}{ }^{11}$ Take $M \in \mathbb{R}$ such that $M>\sup _{n}\left\{\left|\varphi_{n}(s)\right|,|\varphi(s)|,\left|\psi_{n}(s)\right|,|\psi(s)|\right\}$ for all $s \in S$ and $\alpha \in(0,1]$ such that $\alpha M \in[-1,1]$. Then $\alpha \varphi_{n} \succsim_{1} \alpha \psi_{n}, \alpha \varphi_{n} \rightarrow \alpha \varphi$ and $\alpha \psi_{n} \rightarrow \alpha \psi$ so that the continuity of $\succsim_{1}$ implies $\alpha \varphi \succsim_{1} \alpha \psi$. In turn, this is equivalent to $\varphi \succsim_{2} \psi$.

The statement in the theorem now follows from a standard result whose proof we omit:

Lemma $16 \succsim$ is a nontrivial, positively homogeneous, independent, continuous and monotonic preorder on $B(\Sigma)$ if and only if there exists a nonempty, closed and convex set $C$ of probability measures on $\Sigma$ such that

$$
\varphi \succsim \psi \Longleftrightarrow \int_{S} \varphi d P \geq \int_{S} \psi d P \quad \text { for all } P \in C .
$$

Moreover, such $C$ is unique.

Finally, we show that $P(E)=P^{\prime}(E)$ for all $P, P^{\prime} \in C$. Consider $x, y \in X$ such that $u(x)=1$ and $u(y)=0$ and $z \in X$ such that $u(z)=\rho_{E}(E)$. It follows from the representation of Lemma 15 that $x E y \sim z$, and from the representation of Lemma 16 that $P(E)=u(z)=\rho_{E}(E)$ for every $P \in C$.

\section{References}

[1] Frank J. Anscombe and Robert J. Aumann. A definition of subjective probability. Annals of Mathematical Statistics, 34:199-205, 1963.

[2] Truman Bewley. Knightian decision theory: Part I. Technical Report 807, Cowles Foundation, Yale University, 1986.

[3] Ramon Casadesus-Masanell, Peter Klibanoff, and Emre Ozdenoren. Maxmin expected utility over savage acts with a set of priors. Journal of Economic Theory, 92:33-65, 2000.

[4] Ramon Casadesus-Masanell, Peter Klibanoff, and Emre Ozdenoren. Maxmin expected utility through statewise combinations. Economics Letters, 66:49-54, 2000.

[5] Soo Hong Chew and Edi Karni. Choquet expected utility with a finite state space: Commutativity and act-independence. Journal of Economic Theory, 62:469-479, 1994.

[6] Gustave Choquet. Theory of capacities. Annales de l'Institut Fourier (Grenoble), 5:131-295, 1953.

\footnotetext{
${ }^{11}$ As $B(\Sigma)$ is metrizable, it is w.l.o.g. to consider sequences instead of nets.
} 
[7] Paolo Ghirardato, Fabio Maccheroni, and Massimo Marinacci. A fully subjective perspective on ambiguity. Work in progress, Caltech, Università Bocconi and Università di Torino, 2001.

[8] Paolo Ghirardato and Massimo Marinacci. Ambiguity made precise: A comparative foundation. Journal of Economic Theory, forthcoming. http://masada.hss.caltech.edu/ paolo/zi2wp.pdf.

[9] Paolo Ghirardato and Massimo Marinacci. Risk, ambiguity, and the separation of utility and beliefs. Mathematics of Operations Research, forthcoming. http://masada.hss.caltech.edu/ paolo/canonica4wp.pdf.

[10] Itzhak Gilboa. Expected utility with purely subjective non-additive probabilities. Journal of Mathematical Economics, 16:65-88, 1987.

[11] Itzhak Gilboa and David Schmeidler. Maxmin expected utility with a non-unique prior. Journal of Mathematical Economics, 18:141-153, 1989.

[12] Faruk Gul. Savage's theorem with a finite number of states. Journal of Economic Theory, 57:99-110, 1992.

[13] R. Duncan Luce. Utility of Gains and Losses: Measurement-Theoretical and Experimental Approaches. Lawrence Erlbaum, London, 2000.

[14] Mark J. Machina. Almost-objective uncertainty. Mimeo, UC San Diego, July 2001.

[15] Massimo Marinacci. A simple proof of a basic result for multiple priors. Mimeo, University of Toronto, May 1997.

[16] Yutaka Nakamura. Subjective expected utility with non-additive probabilities on finite state spaces. Journal of Economic Theory, 51:346-366, 1990.

[17] John Quiggin. A theory of anticipated utility. Journal of Economic Behavior and Organization, 3:94-101, 1982.

[18] Leonard J. Savage. The Foundations of Statistics. Wiley, New York, 1954.

[19] David Schmeidler. Subjective probability and expected utility without additivity. Econometrica, 57:571-587, 1989.

[20] Lloyd S. Shapley and Manel Baucells. Multiperson utility. Working Paper 779, Department of Economics, UCLA, July 1998.

[21] John von Neumann and Oskar Morgenstern. Theory of Games and Economic Behavior. Princeton University Press, Princeton, second edition, 1947. 